\title{
EXISTENCE OF SOLUTIONS OF FRACTIONAL INTEGRODIFFERENTIAL SYSTEM WITH NONLOCAL CONDITIONS
}

\author{
KAMALENDRA KUMAR ${ }^{1}$ AND RAKESH KUMAR ${ }^{2}$ \\ ${ }^{I}$ Department of Mathematics, S R M S College of Engineering and Technology, \\ Bareilly-243001, India. \\ ${ }^{2}$ Department of Mathematics, Hindu College, Moradabad-244001, India. \\ kamlendra.14kumar@gmail.com; rakeshainil@gmail.com \\ (Received 6 May 2014; accepted 25 February 2015; published on line 29 May 2015)
}

\begin{abstract}
In the present paper we prove the existence and uniqueness of local solutions of a nonlocal Cauchy problem for a class of fractional integrodifferential equation. The results are obtained by using the theory of resolvent operators, the fractional powers of operators, fixed point techniques, and the Gelfand-Shilov principle.
\end{abstract}

ABSTRAK:Menerusi kertas kerja ini, kehadiran dan keunikan penyelesaian lokal terhadap permasalahan tak setempat Cauchy untuk peringkat pecahan persamaan integrodifferential dibuktikan. Keputusan diperolehi menggunakan teori operator leraian, operator kuasa pecahan, teknik titik tetap dan prinsip Gelfand-Shilov.

KEYWORDS: fractional integrodifferential equation; nonlocal conditions; fractional powers; mild and classical solutions; resolvent operators

\section{INTRODUCTION}

The purpose of this paper is to prove the existence and uniqueness of local solutions for nonlocal fractional integrodifferential equations of the form:

$$
\begin{aligned}
& \frac{d^{\alpha} u(t)}{d t^{\alpha}}+A(t) u(t)=f(t, u(t))+\int_{0}^{t} h\left(t, s, u(s), \int_{0}^{s} k(s, \tau, u(\tau)) d \tau\right) d s, \\
& u(0)+g(u)=u_{0},
\end{aligned}
$$

in a Banach space $X$, where $0<\alpha \leq 1, t \geq 0$. Let $J=[0, T]$. We assume that $-A(t)$ is a closed linear operator defined on a dense domain $D(A)$ in $X$ into $X$ such that $D(A)$ is independent of $t$. It is considered also that $-A(t)$ generates an evolution operator in the Banach space $X$. Let $f: J \times X$ into $X, h: J \times J \times X \times X$ into $X, k: J \times J \times X$ into $X$ and $g: C(J, X) \rightarrow D(A)$ be given nonlinear operators.

The differential equations involving fractional derivative in time have recently been proved to be valuable tools in the modeling of many phenomena in various fields of engineering, physics, economy and science. Indeed, we can find numerous applications in viscoelasticity, electrochemistry, porous media, electromagnetic etc. (see [1-6]). They involve a wide area of applications by bringing into a broader paradigm concepts of physics and mathematics [7-9]. Hence, fractional differential equations have become an object of extensive study during recent years as cited in [10-14] and references therein. 
On the other hand, integrodifferential equations arise in many fields such as electronic, fluid dynamics, biological models and chemical kinetics. The equations of basic electric circuit analysis are well-known examples of these equations. Hence, the study of integrodifferential equation is very important. Fractional integrodifferential equations have been studied by many authors [8, 15-18]. Fractional integro-differential equations arise in many fields of engineering such as optimal control problem and heat conduction of materials with memory, etc. The nonlocal condition, which is a generalization of the classical initial condition, was motivated by physical problems. The problem of existence of solutions of evolution equation with nonlocal conditions in Banach space was first studied by Byszewski [19]. As indicated in [19, 20] and the references therein, the nonlocal condition $u(0)+g(u)=u_{0}$ can be applied in physics with better effect than the classical condition $u(0)=u_{0}$.

In the present paper we have generalized the results given by Pazy [21], Balachandran and Chandrasekaran [22] and Debbouche [23]. The rest of this paper is organized as follows: In section 2, we give preliminary results and in section 3, we prove the results of existence and uniqueness of local solutions for the equations (1.1) - (1.2).

\section{PRELIMINARIES}

In this section, we introduce some notations, definitions and results about fractional calculus and resolvent operators. Following Gelfand and Shilov [24], we define the fractional integral of order $\alpha>0$ as

$$
I_{a}^{\alpha} f(t)=\frac{1}{\Gamma(\alpha)} \int_{a}^{t}(t-s)^{\alpha-1} f(s) d s,
$$

and also, the fractional derivative of the function $f$ of order $0<\alpha<1$

$$
{ }_{a} D_{t}^{\alpha} f(t)=\frac{1}{\Gamma(1-\alpha)} \frac{d}{d t} \int_{a}^{t} f(s)(t-s)^{-\alpha} d s,
$$

where $f$ is an abstract continuous function on the interval $[a, b]$ and $\Gamma(\alpha)$ is the Gamma function, see [25].

Definition 2.1: By a solution of (1.1) - (1.2), we mean a function $u$ with values in $X$ such that:

(1) $u$ is continuous function on $J$ and $u(t) \in D(A)$,

(2) $\frac{d^{\alpha} u}{d t^{\alpha}}$ exists and is continuous on $(0, T), 0<\alpha<1$, and $u$ satisfies $(1.1)$ on $(0, T)$ and the nonlocal condition (1.2).

Definition 2.2: (See [26]) A resolvent operator for problem (1.1) - (1.2) is a bounded operator-valued function $R(t, s) \in B(X), 0 \leq s \leq t \leq T$, having the following properties:

(a) $R(t, s)$ is strongly continuous in $s$ and $t, R(s, s)=I$, the identity operator on $X$, $0 \leq s \leq T$, and $\|R(t, s)\| \leq M e^{\beta(t-s)}$ for some constants $M$ and $\beta$.

(b) $R(t, s) E \subset E, R(t, s)$ is strongly continuous in $s$ and $t$ on $E$, and $E$ is the Banach space formed from $D(A)$, the domain $-A(t)$, endowed with the graph norm.

(c) For every $x \in X, R(t, s) x$ is continuously differentiable in $s \in J$ and 


$$
\frac{\partial R}{\partial s}(t, s) x=R(t, s) A(s) x .
$$

(d) For every $x \in X$, and $s \in J, R(t, s) x$ is continuously differentiable in $t \in J$ and

$$
\frac{\partial R}{\partial t}(t, s) x=-A(t) R(t, s) x
$$

with $\frac{\partial R}{\partial s}(t, s) x$ and $\frac{\partial R}{\partial t}(t, s) x$ are strongly continuous on $0 \leq s \leq t \leq T$. Here, $R(t, s)$ can be deduced from the evolution operator of the generator $-A(t)$. The resolvent operator is similar to the evolution operator for non-autonomous differential equations in Banach space. It will not, however, be an evolution operator because it will not satisfy an evolution or semigroup property. Because a number of results follow directly from the definition of resolvent operator.

Definition 2.3: A continuous solution $u$ : $J \rightarrow X$ is said to be a mild solution of problem (1.1) $-(1.2)$ on $J$ if for all $u_{0} \in X$, it satisfies the following integral equation

$$
\begin{gathered}
u(t)=R(t, 0)\left[u_{0}-g(u)\right]+\frac{1}{\Gamma(\alpha)} \\
\int_{0}^{t}(t-s)^{\alpha-1} R(t, s)\left[f(s, u(s))+\int_{0}^{s} h\left(s, \tau, u(\tau), \int_{0}^{\tau} k(\tau, \mu, u(\mu)) d \mu\right) d \tau\right] d s .
\end{gathered}
$$

We define the fractional power $A^{-q}(t)$ by

$$
A^{-q}(t)=\frac{1}{\Gamma(q)} \int_{0}^{\infty} x^{q-1} R(x, t) d x, q>0 .
$$

For $0<q \leq 1, A^{q}(t)$ is a closed linear operator whose domain $D\left(A^{q}\right) \supset D(A)$ is dense in $X$. The closedness of $A^{q}$ implies that $D\left(A^{q}\right)$, endowed with the graph norm of $A^{q}, \quad\|u\|_{D(A)}=\|u\|+\left\|A^{q} u\right\|, u \in D\left(A^{q}\right)$, is a Banach space. Since $0 \in \rho(A), A^{q}$ is invertible. Its graph norm I\|. $\| I$ is equivalent to the norm $\|u\|_{q}=\left\|A^{q} u\right\|$. Thus $D\left(A^{q}\right)$ equipped with the norm $\|.\|_{q}$ is a Banach space, which we denote by $X_{q}$.

\section{EXISTENCE THEOREM}

To prove the main results we state the following lemma:

Lemma 3.1: (see [21, section 2.6]) Let $A(t)$ be the infinitesimal generator of a resolvent operator $R(t, s)$. We denote by $\rho[A(t)]$ the resolvent set of $A(t)$. If $0 \in \rho[A(t)]$, then

(a) $R(t, s): X \rightarrow D\left(A^{q}\right)$ for every $0 \leq s \leq t \leq T$ and $q \geq 0$,

(b) For every $u \in D\left(A^{q}\right)$, we have $R(t, s) A^{q}(t) u=A^{q}(t) R(t, s) u$,

(c) The operator $A^{q} R(t, s)$ is bounded and $\left\|A^{q} R(t, s)\right\| \leq M_{q, \beta}(t-s)^{-q}$.

Theorem 1.1: Assume that

(a) $-A(t)$ is the infinitesimal generator of a resolvent operator $R(t, s), 0 \leq s \leq t \leq T$, in $X$.

(b) $0 \in \rho[-A(t)]$, the resolvent set.

(c) For $q \geq 0$, the fractional power $A^{q}$ satisfies $\left\|A^{q} R(t, s)\right\| \leq M_{q, \beta}(t-s)^{-q}$ for $0 \leq$ $s \leq t \leq T$, where $M_{q, \beta}$ is a real constant. 
(d) For an open subset $P$ of $J \times X_{q}, f: P \rightarrow X$ satisfies the condition, if for every $(t, u) \in P$ there is a neighborhood $V \subset P$ and constants $L \geq 0,0<\vartheta \leq 1$, such that

$$
\left\|f\left(t_{1}, u_{1}\right)-f\left(t_{2}, u_{2}\right)\right\| \leq L\left(\left|t_{1}-t_{2}\right| \vartheta+\left\|u_{1}-u_{2}\right\|_{q}\right)(3.1)
$$

for all $\left(t_{i}, u_{i}\right) \in V, i=1,2$.

(e) For an open subset Q of $J \times J \times X_{q} \times X_{q}, h: Q \rightarrow X$ satisfies the condition, if for every $(t, s, u, v) \in Q$ there is a neighborhood $U \subset Q$ and constants $L_{1} \geq 0,0<\vartheta \leq$ 1 , such that

$$
\begin{aligned}
& \left\|h\left(t_{1}, s_{1}, u_{1}, v_{1}\right)-h\left(t_{2}, s_{2}, u_{2}, v_{2}\right)\right\| \\
& \leq L_{1}\left(\left|t_{1}-t_{2}\right|^{\vartheta}+\left|s_{1}-s_{2}\right|^{\vartheta}+\left\|u_{1}-u_{2}\right\|_{q}+\left\|v_{1}-v_{2}\right\|_{q}\right)
\end{aligned}
$$

for all $\left(t_{i}, s_{i}, u_{i}, v_{i}\right) \in U, i=1,2$.

(f) For an open subset $\mathrm{R}$ of $J \times J \times X_{q}, k: R \rightarrow X$ satisfies the condition, if for every $(t, s, u) \in R$ there is a neighborhood $W \subset R$ and constants $L_{2} \geq 0,0<\vartheta \leq 1$, such that

$\left\|k\left(t_{1}, s_{1}, u_{1}\right)-k\left(t_{2}, s_{2}, u_{2}\right)\right\| \leq L_{2}\left(\left|t_{1}-t_{2}\right|^{\vartheta}+\left|s_{1}-s_{2}\right| \vartheta+\left\|u_{1}-u_{2}\right\|_{q}\right)$

for all $\left(t_{i}, s_{i}, u_{i}\right) \in W, i=1,2$.

(g) $g: Y \rightarrow X_{q}$ is continuous and there exists a number $b$ such that $\|R(t, 0)\|<\frac{1}{2 b}$ and

$\|g(x)-g(y)\|_{q} \leq b\|x-y\|_{\infty}$

for all $x, y \in Y$. Note that if $z \in Y$, then $A^{-q} z \in Y$.

Then the problem (1.1) - (1.2) has a unique local solution $u \in C([0, T): X) \cap$ $C^{1}((0, T): X)$.

Proof: Choose $t^{*}>0$ and $\delta>0$ such that estimates (3.1) - (3.4) hold on the sets

$$
V=\left\{(t, u): 0 \leq t \leq t^{*},\left\|u-u_{0}\right\| \leq \delta\right\}
$$

$$
U=\left\{(t, s, u, v): 0 \leq t, s \leq t^{*},\left\|u-u_{0}\right\| \leq \delta,\left\|v-v_{0}\right\| \leq \delta\right\},
$$

and $\quad W=\left\{(t, s, u): 0 \leq t, s \leq t^{*},\left\|u-u_{0}\right\| \leq \delta\right\}$, respectively.

Let $B=\max _{0 \leq t<T}\left\|f\left(t, u_{0}\right)\right\|$

and

$$
H=\max _{0 \leq t, s \leq t^{*}}\left\|h\left(t, s, u_{0}, \int_{0}^{s} k\left(s, \tau, u_{0}\right) d \tau\right)\right\|
$$

Set $\lambda=\sup _{x \in Y}\|g(x)\|_{q}$ and choose $T$ such that for $0 \leq t<T$,

and

$$
\|R(t, 0)-I\|\left[\left\|u_{0}\right\|_{q}+\lambda\right] \leq \frac{\delta}{2}, \quad 0 \leq t<T
$$

$$
0<T<\min \left\{t^{*},\left[\frac{\delta}{2} M_{q, \beta}^{-1} \Gamma(\alpha)(\alpha-q)\left(L \delta+B+L_{1} \delta T+L_{1} L_{2} \delta T^{2}+H T\right)^{-1}\right]^{\frac{1}{\alpha-q}}\right\}
$$

Let $Y=C((0, T]: X)$ be endowed with the supremum norm

$$
\|y\|_{\infty}=\sup _{0 \leq t<T}\|y(t)\|_{q}, \quad y \in Y .
$$


Then $Y$ be a Banach space. Define a map $F: Y \rightarrow Y$

$$
\begin{gathered}
F y(t)=R(t, 0) A^{q}\left[u_{0}-g\left(A^{-q} y\right)\right]+\frac{1}{\Gamma(\alpha)} \int_{0}^{t}(t-s)^{\alpha-1} A^{q} R(t, s) f\left(s, A^{-q} y(s)\right) d s \\
+\frac{1}{\Gamma(\alpha)} \int_{0}^{t}(t-s)^{\alpha-1} A^{q} R(t, s) \int_{0}^{s} h\left(s, \tau, A^{-q} y(\tau), \int_{0}^{\tau} k\left(\tau, \mu, A^{-q} y(\mu)\right) d \mu\right) d \tau d s .
\end{gathered}
$$

Obviously, $F y(0)=A^{q}\left[u_{0}-g\left(A^{-q} y\right)\right]$. Let $S$ be the nonempty closed and bounded subset of $Y$ defined by

$$
S=\left\{y: y \in Y, y(0)=A^{q}\left[u_{0}-g\left(A^{-q} y\right)\right],\left\|y(t)-A^{q}\left[u_{0}-g\left(A^{-q} y\right)\right]\right\| \leq \delta\right\} .
$$

For $y \in S$, we have

$$
\begin{aligned}
& \left\|F y(t)-A^{q}\left[u_{0}-g\left(A^{-q} y\right)\right]\right\| \leq\|R(t, 0)-I\|\left\|A^{q}\left[u_{0}-g\left(A^{-q} y\right)\right]\right\| \\
& +\frac{1}{\Gamma(\alpha)} \int_{0}^{t}(t-s)^{\alpha-1}\left\|A^{q} R(t, s)\right\|\left\|f\left(s, A^{-q} y(s)\right)-f\left(s, u_{0}\right)\right\| d s \\
& +\frac{1}{\Gamma(\alpha)} \int_{0}^{t}(t-s)^{\alpha-1}\left\|A^{q} R(t, s)\right\|\left\|f\left(s, u_{0}\right)\right\| d s \\
& +\frac{1}{\Gamma(\alpha)} \int_{0}^{t}(t-s)^{\alpha-1}\left\|A^{q} R(t, s)\right\| \|\left[\int_{0}^{s} h\left(s, \tau, A^{-q} y(\tau), \int_{0}^{\tau} k\left(\tau, \mu, A^{-q} y(\mu)\right) d \mu\right) d \tau\right. \\
& \left.-\int_{0}^{s} h\left(s, \tau, u_{0}, \int_{0}^{\tau} k\left(\tau, \mu, u_{0}\right) d \mu\right) d \tau\right] \| d s \\
& +\frac{1}{\Gamma(\alpha)} \int_{0}^{t}(t-s)^{\alpha-1}\left\|A^{q} R(t, s)\right\|\left\|\int_{0}^{s} h\left(s, \tau, u_{0}, \int_{0}^{\tau} k\left(\tau, \mu, u_{0}\right) d \mu\right) d \tau\right\| d s \\
& \leq \frac{\delta}{2}+\frac{M_{q, \beta}}{\Gamma(\alpha)}(\alpha-q)^{-1} T^{\alpha-q}(L \delta+B)+\frac{M_{q, \beta}}{\Gamma(\alpha)}(\alpha-q)^{-1} T^{\alpha-q}\left\{L_{1}\left(\delta+L_{2} \delta T\right) T\right\} \\
& +\frac{M_{q, \beta}}{\Gamma(\alpha)}(\alpha-q)^{-1} T^{\alpha-q} H T \\
& \leq \frac{\delta}{2}+\frac{M_{q, \beta}}{\Gamma(\alpha)}(\alpha-q)^{-1} T^{\alpha-q}\left[L \delta+B+L_{1} \delta T+L_{1} L_{2} \delta T^{2}+H T\right] \leq \frac{\delta}{2}+\frac{\delta}{2}=\delta .
\end{aligned}
$$

Therefore, $F$ maps $S$ into itself. Moreover, if $y_{1}, y_{2} \in S$, then

$$
\begin{gathered}
\left\|F y_{1}(t)-F y_{2}(t)\right\| \leq\|R(t, 0)\|\left\|g\left(A^{-q} y_{1}\right)-g\left(A^{-q} y_{2}\right)\right\|_{q} \\
+\frac{1}{\Gamma(\alpha)} \int_{0}^{t}(t-s)^{\alpha-1}\left\|A^{q} R(t, s)\right\|\left\|f\left(s, A^{-q} y_{1}(s)\right)-f\left(s, A^{-q} y_{2}(s)\right)\right\| d s \\
+\frac{1}{\Gamma(\alpha)} \int_{0}^{t}(t-s)^{\alpha-1}\left\|A^{q} R(t, s)\right\| \| \int_{0}^{s} h\left(s, \tau, A^{-q} y_{1}(\tau), \int_{0}^{\tau} k\left(\tau, \mu, A^{-q} y_{1}(\mu)\right) d \mu\right) \\
-\int_{0}^{s} h\left(s, \tau, A^{-q} y_{2}(\tau), \int_{0}^{\tau} k\left(\tau, \mu, A^{-q} y_{2}(\mu)\right) d \mu\right) d \tau \| d s
\end{gathered}
$$




$$
\begin{gathered}
\leq b\|R(t, 0)\|\left\|y_{1}-y_{2}\right\|_{\infty}+\frac{1}{\Gamma(\alpha)} M_{q, \beta} T^{\alpha-q}(\alpha-q)^{-1} L\left\|y_{1}-y_{2}\right\|_{Y} \\
+\frac{1}{\Gamma(\alpha)} M_{q, \beta} T^{\alpha-q}(\alpha-q)^{-1} L_{1}\left[\left(\left\|y_{1}-y_{2}\right\|_{Y}+L_{2}\left\|y_{1}-y_{2}\right\|_{Y} T\right) T\right] \\
\leq b\|R(t, 0)\|\left\|y_{1}-y_{2}\right\|_{Y}+\frac{1}{\Gamma(\alpha)} M_{q, \beta} T^{\alpha-q}(\alpha-q)^{-1}\left[L+L_{1}\left(1+L_{2} T\right) T\right]\left\|y_{1}-y_{2}\right\|_{Y} \\
\leq \frac{1}{2}\left\|y_{1}-y_{2}\right\|_{Y}+\frac{1}{2}\left\|y_{1}-y_{2}\right\|_{Y}, \\
\text { which implies that }\left\|F y_{1}-F y_{2}\right\| \leq \frac{1}{2}\left\|y_{1}-y_{2}\right\|_{Y}+\frac{1}{2}\left\|y_{1}-y_{2}\right\|_{Y} .
\end{gathered}
$$

By the contraction mapping theorem, mapping $F$ has a unique fixed point $y \in S$. This fixed point satisfies the integral equation

$$
\begin{gathered}
y(t)=R(t, 0) A^{q}\left[u_{0}-g\left(A^{-q} y\right)\right]+\frac{1}{\Gamma(\alpha)} \int_{0}^{t}(t-s)^{\alpha-1} A^{q} R(t, s) f\left(s, A^{-q} y(s)\right) d s \\
+\frac{1}{\Gamma(\alpha)} \int_{0}^{t}(t-s)^{\alpha-1} A^{q} R(t, s) \int_{0}^{s} h\left(s, \tau, A^{-q} y(\tau), \int_{0}^{\tau} k\left(\tau, \mu, A^{-q} y(\mu)\right) d \mu\right) d \tau d s .
\end{gathered}
$$

From (3.1), (3.2) and the continuity of $y$ it follow that

$$
t \rightarrow f\left(t, A^{-q} y(t)\right)
$$

and

$$
t \rightarrow h\left(t, s, A^{-q} y(s), \int_{0}^{s} k\left(s, \tau, A^{-q} y(\tau)\right) d \tau\right)
$$

are continuous on $[0, T]$, and, hence, there exist constants $N_{1}$ and $N_{2}$ such that

$$
\left\|f\left(t, A^{-q} y(t)\right)\right\| \leq N_{1}
$$

and

$$
\left\|h\left(t, s, A^{-q} y(s), \int_{0}^{s} k\left(s, \tau, A^{-q} y(\tau)\right) d \tau\right)\right\| \leq N_{2}
$$

By using the same method as in [20, Theorem 3.2], we can prove that $y(t)$ is locally Hölder continuous on $(0, T]$. Then there exist a constant $C$ such that for every $t^{\prime}>0$, we have

$$
\|y(t)-y(s)\| \leq C \mid t-s^{\gamma}
$$

for all $0 \leq t^{\prime} \leq t, s \leq T$. The local Hölder continuity of $t \rightarrow f\left(t, A^{-q} y(t)\right)$ follows from

$$
\begin{aligned}
\left\|f\left(t, A^{-q} y(t)\right)-f\left(s, A^{-q} y(s)\right)\right\| \leq L\left(|t-s|^{\vartheta}\right. & +\|y(t)-y(s)\|) \\
& \leq C_{1}\left(|t-s|^{\vartheta}+|t-s|^{\gamma}\right)
\end{aligned}
$$

for some $C_{1}>0$ and the local Hölder continuity of

follows from

$$
t \rightarrow h\left(t, s, A^{-q} y(s), \int_{0}^{s} k\left(s, \tau, A^{-q} y(\tau)\right) d \tau\right)
$$




$$
\begin{gathered}
\left\|h\left(t, s, A^{-q} y(s), \int_{0}^{s} k\left(s, \tau, A^{-q} y(\tau)\right) d \tau\right)-h\left(t, \mu, A^{-q} y(\mu), \int_{0}^{s} k\left(\mu, \phi, A^{-q} y(\phi)\right) d \phi\right)\right\| \\
\leq L_{1}\left\{|s-\mu|^{\vartheta}+\|y(s)-y(\mu)\|+L_{2}\left(|s-\mu|^{\vartheta}+|\tau-\phi|^{\vartheta}+\|y(\tau)-y(\phi)\|\right) T\right\} \\
\leq L_{1}\left\{|s-\mu|^{\vartheta}+|s-\mu|^{\gamma}+L_{3}\left(|s-\mu|^{\vartheta}+|\tau-\phi|^{\vartheta}+|\tau-\phi|^{\gamma}\right) T\right\}
\end{gathered}
$$

for some $L_{3}>0$. Let $y$ be a solution of (3.7). Consider the inhomogeneous initial value problem

$$
\begin{aligned}
& \frac{d^{\alpha} u(t)}{d t^{\alpha}}+A(t) u(t) \\
& \quad=f\left(t, A^{-q} y(t)\right)+\int_{0}^{t} h\left(t, s, A^{-q} y(s), \int_{0}^{s} k\left(s, \tau, A^{-q} y(\tau)\right) d \tau\right) d s \\
& u(0)+g\left(A^{-q} y\right)=u_{0}
\end{aligned}
$$

This problem has a unique solution $u \in C^{1}((0, T]: X)$ [21], which is given by

$$
\begin{gathered}
u(t)=R(t, 0)\left[u_{0}-g\left(A^{-q} y\right)\right]+\frac{1}{\Gamma(\alpha)} \int_{0}^{t}(t-s)^{\alpha-1} R(t, s) f\left(s, A^{-q} y(s)\right) d s \\
+\frac{1}{\Gamma(\alpha)} \int_{0}^{t}(t-s)^{\alpha-1} R(t, s) \int_{0}^{s} h\left(s, \tau, A^{-q} y(\tau), \int_{0}^{\tau} k\left(\tau, \mu, A^{-q} y(\mu)\right) d \mu\right) d \tau d s .
\end{gathered}
$$

for $t>0$, each term of (3.12) belongs to $D(A)$ and a fortiori in $D\left(A^{q}\right)$. Operating on both sides of (3.12) with $A^{q}$ we find that

$$
\begin{gathered}
A^{q} u(t)=R(t, 0) A^{q}\left[u_{0}-g\left(A^{-q} y\right)\right]+\frac{1}{\Gamma(\alpha)} \int_{0}^{t}(t-s)^{\alpha-1} A^{q} R(t, s) f\left(s, A^{-q} y(s)\right) d s \\
+\frac{1}{\Gamma(\alpha)} \int_{0}^{t}(t-s)^{\alpha-1} A^{q} R(t, s) \int_{0}^{s} h\left(s, \tau, A^{-q} y(\tau), \int_{0}^{\tau} k\left(\tau, \mu, A^{-q} y(\mu)\right) d \mu\right) d \tau d s .
\end{gathered}
$$

From (3.7) the right hand side of (3.13) equals $y(t)$ and therefore $u(t)=A^{-q} y(t)$ and by (3.12), $\mathrm{u}$ is a $\mathrm{C}^{1}((0, \mathrm{~T}]: \mathrm{X})$ solution of $(1.1)-(1.2)$. The uniqueness of $\mathrm{u}$ follows from the uniqueness of the solutions of (3.7) and (3.10)-(3.11). Hence, the theorem is proved.

\section{CONCLUSION}

In this paper, the existence and uniqueness of mild and classical solutions for the nonlinear fractional integrodifferential equation with nonlocal condition are discussed. We applied the resolvant operators, the fractional powers of operaters, fixed point technique and Gelfand-Shilov principle to establish the existence results. The results presented in this paper may be useful in the field of engineering and physics.

\section{ACKNOWLEDGEMENT}

The authors wish to thank the referees for their valuable comments and suggestions. 


\section{REFERENCES}

[1] Mainardi F. (1997) Fractional calculus: Some basic problems in continuum and statistical mechanics, in Fractals and Fractional Calculus in Continuum Mechanics, Carpinteri A,Mainardi F, eds.), Springer-Verlag, Wien, pp. 291-348.

[2] Metzler F, Schick W, Kilan HG, Nonnenmacher TF. (1995) Relaxation in filled polymers: A fractional calculus approach. Journal of Chemical Physics, 103:7180-7186.

[3] Diethelm K, Freed AD. (1999) On the solution of nonlinear fractional order differential equations used in the modeling of viscoelasiticity, Scientific Computing in Chemical Engineering II- Computational Fluid Dynamics, Reaction Engineering and Molecular Properties (Keil F, Mackens W, Voss H, Werther J., eds) Springer-Verlag, Heidelberg, pp. 217-224.

[4] Gaul L, Klien P,Kempfle S. (1991) Damping description involving fractional operators. Mechanical Systems and Signal Processing, 5:81-88.

[5] Hilfer R. (2000) Application of fractional calculus in physics, World Scientific, Singapore.

[6] GlockleWG, Nonnenmacher TF. (1995) A fractional calculus approach of self-similar protein dynamics. Biophysical Journal, 68:46-53.

[7] Baleanu D, Trujillo JJ. (2010) A new method of finding the fractional Euler-Lagrange and Hamilton equations within Caputo fractional derivatives. Commun. Nonlinear Sci. Numer. Simul., 15(5):1111-1115.

[8] Balachandran K, Trujillo JJ. (2010) The nonlocal Cauchy problem for nonlinear fractional Integro-differential equations in Banach spaces. Nonlinear Analysis: Theory, Methods and Applications, 72:4587-4593.

[9] Agrawal RP, Lakshmikantham V, Nieto JJ. (2010) On the concept of solution for fractional differential equations with uncertainty. Nonlinear Anal., 72:2859-2862.

[10] Li F. (2010) Mild solutions for fractional differential equations with nonlocal conditions. Adv. Difference Equations, Article ID 287861, 9 pages.

[11] Mophou GM, Guerekata GMN. (2009) Mild solutions for semilinear fractional differential equations. Electron. J. Differential Equations, 21:1-9.

[12] El-Borai MM. (2002) Some probability densities and fundamental solutions of fractional evolutions equations. Chaos, Solutions and Fractals, 14(3):433-440.

[13] El-Borai MM. (2005) On some fractional evolution equations with nonlocal conditions. Int. J. Pure Appl. Math.,24(3):405-413.

[14] El-Borai MM. (2004) Semigroups and some nonlinear fractional differential equations. Appl. Math. Comput.,149(3):823-831.

[15] Li F, Guerekata GMN. (2010) Existence and uniqueness of mild solution for fractional integro-differential equations. Adv. Difference Equations, doi: 1155/2010/158789 (available on line).

[16] Ahmed HM. (2009) Semilinear differential equations of fractional order with nonlocal conditions. Int. J. of Appl. Math. Mech., 5(3):69-74.

[17] Bragdi M, Hazi M. (2010) Existence and controllability result for an evolution fractional integro-differential system. Int. J. Contemp. Math. Sciences, 5(19):901-910.

[18] El-Borai MM, Debbouche A. (2009) On some fractional integro-differential equations with analytic semigroups. Int. J. Contemp. Math. Sciences, 4(28):1361-1371.

[19] Byszewski L. (1991) Theorems about the existence and uniqueness of solutions of a semilinear evolution nonlocal Cauchy problem. J. Math. Anal. Appl., 162:494-505.

[20] Deng K. (1993) Exponential decay of solutions of semilinear parabolic equations with nonlocal conditions. J. Math. Anal. Appl., 179:630-637.

[21] Pazy A. (1983) Semigroups of linear operators and applications to partial differential equations, Springer-Verlag, New York, 1983.

[22] Balachandran K, Chandrasekaran M. (1997) Existence of solutions of nonlinear integrodifferential equation with nonlocal condition. Journal of Applied Mathematics and Statistics Analysis, 10(3):279-288.

[23] Debbouche A. (2010) Fractional evolution integro-differential systems with nonlocal conditions. Advances in Dynamical Systems and Applications,5:49-60. 
[24] Gelfand IM, Shilov GE. (1959) Generalized functions. vol. 1, Moscow, Nauka.

[25] Debbouche A, El-Borai MM. (2009) Weak almost periodic and optimal mild solutions of fractional evolution equations. Electron. J. Differential Equations, 46:1-8.

[26] Tidke HL, Dhakne MB. (2011) Existence of solutions and controllability of nonlinear mixed integrodifferential equation with nonlocal condition. Applied Mathematics E-Notes, 11:1222.

AMS Subject Classification: 34G20, 34K05, 26A33, 35B65, 35A07. 